\title{
Recommending inclusion of HFE C282Y homozygotes in the ACMG actionable gene list: cop-out or stealth move toward population screening?
}

\author{
Anne-Marie Laberge, $\mathrm{MD}, \mathrm{PhD}^{1,2}$
}

Hereditary hemochromatosis ( $\mathrm{HH})$ has long been considered a preventable disease because its complications are a result of progressive iron overload and can be effectively treated with phlebotomies. Before the discovery of the HFE gene, prevention relied on detection via iron measures in blood (transferrin, ferritin) and liver biopsy in individuals with liver disease or family history. The discovery of the HFE gene in 1996 raised hopes of identifying individuals at risk of $\mathrm{HH}$ early and accurately. ${ }^{1}$ In 1997, the Centers for Disease Control and Prevention convened an expert conference to examine the implications of the discovery of the HFE gene for population screening. ${ }^{2}$ Experts did not recommend population-based genetic screening for $\mathrm{HH}$ at that time, due to uncertainties about the prevalence of HFE variants, the penetrance of these variants, and the performance of genotype-based tests compared with phenotypic tests (transferrin saturation, ferritin). Over the following years, new studies answered some of these questions. The prevalence of C282Y homozygotes was shown to be about $0.26 \%$ in adult members of the Kaiser Permanente care program in San Diego, California. ${ }^{3}$ A US study reported an estimated clinical penetrance of $1 \%$ in C282Y homozygotes but was criticized for using a restrictive definition of disease, i.e., presence of multiple signs of hemochromatosis in the same individual (chronic fatigue, joint symptoms, skin darkening, impotence, diabetes, etc.), leading to an overly conservative estimate of penetrance. ${ }^{4}$ In fact, individuals may develop only some of the complications of iron overload but may still benefit from preventive treatment. In addition this study enrolled a population with a large age range, from about 25 to 80 years of age; penetrance is expected to be low in younger adults, which could have also led to an underestimation of clinically relevant penetrance. Despite criticisms about the methodology used, the low penetrance reported in this study cooled interest in population-based screening for hereditary hemochromatosis in the United States.

Since then, further studies of prevalence, penetrance, and screening strategies have helped clarify the natural history of the disease and the potential impacts of screening. In this issue, Grosse et al. $^{5}$ review the available evidence from population-based studies on the cumulative risk of severe liver disease in male HFE C282Y homozygotes. Using four cohort studies, they estimate the pooled prevalence of severe liver disease among male homozygotes over 60 years of age to be $9.0 \%(2.6-15.3 \%)$. As the authors note, the use of crosssectional prevalence of clinical disease as a proxy for penetrance (as in the 2002 penetrance study) can be misleading. ${ }^{4,5}$ Their new estimate, based on cumulative incidence of disease, offers a more reliable estimate of penetrance. In addition, it is applicable to the population most at risk of developing complications, i.e., older males, thereby providing relevant evidence for decisions about screening for $\mathrm{HH}$.

This new penetrance estimate for $\mathrm{HH}$ in male $\mathrm{C} 282$ homozygotes opens the door to a new discussion about population-based screening for $\mathrm{HH}$, at least in this particular population.

The application of the World Health Organization population screening criteria to general population screening for hereditary hemochromatosis was generally favorable when assessed in 1998, but the evidence at that time was lacking about natural history and agreement on whom to treat. ${ }^{2}$ Evidence was also still lacking about cost, positive predictive value, and acceptability of genotype-based screening. How close are we now to meeting these criteria? Our knowledge of natural history has improved, as demonstrated with the evidence provided by the studies analyzed by Grosse et al. ${ }^{5}$ Guidelines exist on whom to treat, although there seems to be disagreement among guidelines. ${ }^{6,7}$ Cost of genotyping has dramatically decreased over the last two decades, and costeffectiveness studies have been supportive of $\mathrm{HH}$ screening, at least in at-risk populations. ${ }^{8}$ Population screening studies have shown genotype-based screening to be acceptable. ${ }^{9}$ Why not use the estimate of cumulative incidence of liver disease offered by Grosse et al., ${ }^{5}$ i.e., penetrance of liver disease in $\mathrm{C} 282 \mathrm{Y}$ homozygotes in males, to argue in favor of populationbased screening in males? The frequency of C282Y homozygotes (1/300 in non-Hispanic whites) yields an estimate of

${ }^{1}$ Department of Pediatrics, Université de Montréal, Montreal, Quebec, Canada; ${ }^{2}$ Medical Genetics and Research Center, CHU Sainte-Justine, Montreal, Quebec, Canada. Correspondence: Anne-Marie Laberge (anne-marie.laberge.hsj@ssss.gouv.qc.ca)

Submitted 8 August 2017; accepted 16 August 2017; advance online publication 19 October 2017. doi:10.1038/gim.2017.161 
approximately 1 million at-risk individuals in the US population, about 100,000 of whom will develop meaningful disease based on the estimate of $9 \%$ penetrance by Grosse et al. ${ }^{5}$ The disorder has a recognizable latent or minimally symptomatic phase and an easily accessible, acceptable treatment, known to reverse manifestations of the disease when started early enough.

The question remains whether the most appropriate screening test is genotype-based screening or phenotypic screening using iron overload biochemical measures. Genotype-based tests are highly specific, but individuals with the risk genotype may not have iron overload, or at least not yet. Genotype-based screening allows for primary prevention of iron overload, but requires follow-up of all HFE C282Y homozygotes to prevent liver disease in 9\%. HFE C282Y homozygotes identified through genetic screening will need to be assessed using biochemical tests to look for biochemical signs of iron overload. Biochemical tests (transferrin saturation, ferritin) are more sensitive, and will detect individuals who have iron overload from non-HFE related causes and could potentially benefit from treatment. This is especially true in nonwhite populations. For this reason, biochemical screening may be more useful to identify individuals who are more likely to get liver disease or other complications of $\mathrm{HH}$ and therefore more likely to benefit from screening in the short term.

There also remains the question of screening benefit for women. Female C282Y homozygotes experience lower penetrance than males but are not exempt from developing complications, including liver disease. Thus, it would be relevant to estimate penetrance in older women, to inform future policies on genetic screening in the population as a whole.

Based on their estimated cumulative disease incidence of liver disease of $9 \%$ in males, paired with available evidence on management of presymptomatic patients, Grosse et al. ${ }^{5}$ suggest that "a potentially effective strategy for increasing the early detection and prevention of clinical iron overload and severe disease is to include HFE C282Y homozygosity in lists of medically actionable gene variants when reporting results from genome or exome sequencing." Since the use of next-generation sequencing and whole-exome/whole-genome sequencing has become clinically available, the discussion around actionable incidental findings has been active in the genetics community. The American College of Medical Genetics and Genomics (ACMG) has issued recommendations about disclosure first in 2013, with the latest update in 2017. ${ }^{10,11}$ HFE C282Y is a variant that has been studied extensively to assess its penetrance, its genotype-phenotype correlation, and the impacts of treatment in homozygotes. Yet, despite a great deal of evidence, it is not on the ACMG actionable gene list or a target for screening, while we actually have less evidence regarding penetrance, genotype-phenotype correlation, or impacts of preventive or treatment measures for many of the specific pathogenic gene variants in the genes currently on the ACMG actionable gene list. The HFE C282Y homozygote status is actionable. Is its absence on the current list explained by what is perceived as its low penetrance? Is this variant a victim of the fact that we know enough about it to know the limits of its predictive value?

Given the prevention opportunity, including HFE C282Y homozygosity in the ACMG actionable gene list is justifiable, but it is not the most efficient approach to reduce morbidity of $\mathrm{HH}$ from a public health perspective. Only a minority of individuals in the general population will get such tests in a clinical setting and rarely would healthy adults undergo genome-scale sequencing, begging the question of direct ascertainment through primary screening.

The evidence we have about returning HFE C282Y status as a secondary finding comes mainly from the Hemochromatosis and Iron Overload Screening (HEIRS) study and from the Electronic Medical Records and Genomics (eMERGE) Network. $^{12,13}$ The HEIRS study found that symptoms attributable to HFE C282Y homozygosity were no more common in individuals identified by population screening than in control subjects. ${ }^{12}$ Genetic testing was well accepted, but participants with normal HFE test results understood their results and recommendations better than those with one or two mutations. ${ }^{14}$ They concluded that they would not recommend generalized population screening in a primary care population as performed in their study, but that there could be a role for focused screening in Caucasian men. ${ }^{12}$ Initially, the eMERGE Network found mixed views from researchers about returning findings of HFE gene mutations associated with hemochromatosis due to low penetrance. ${ }^{13} \mathrm{~A}$ follow-up study found high diagnostic rates of $\mathrm{HH}$ in male and female $\mathrm{C} 282 \mathrm{Y}$ homozygotes $(24.4 \%$ and $14.0 \%$ respectively) in the eMERGE cohort. ${ }^{15}$ They also concluded that reporting HFE $\mathrm{C} 282 \mathrm{Y}$ homozygote status as an incidental finding should be considered.

Is the recommendation to include HFE C282Y homozygosity on the ACMG actionable gene list a consolation prize for a condition once thought to be a prime candidate for population-based screening? Or is it a first step back toward population-based screening in a target group? Even in the age of genomic medicine, we must remember that improvements in population health will only be achieved if genetic findings are used to improve management of individuals at risk. Inclusion in the ACMG actionable gene list is easy to implement: it does not entail additional costs for screening (except for downstream costs for identified individuals), and hopefully improves health outcomes-albeit for a very small number of individuals. Recommending targeted screening of older males for HFE C282Y homozygosity is not as simple to implement: it requires uptake by health professionals, access to testing, and access to appropriate management. On the other hand, it would have a greater impact on health outcomes related to $\mathrm{HH}$ in the general population than inclusion of HFE C282Y homozygosity in the ACMG actionable gene list. Therefore, perhaps the question of inclusion on the ACMG list is the easy one, and the real question that remains is whether the time has come to 
reconsider targeted primary screening and to call for focused studies on outcomes of targeted screening for $\mathrm{HH}$ and the best way to implement it.

\section{DISCLOSURE}

The author declares no conflict of interest.

\section{REFERENCES}

1. Feder JN, Gnirke A, Thomas W, et al. A novel MHC class I-like gene is mutated in patients with hereditary haemochromatosis. Nat Genet. 1996;13:399-408.

2. Burke W, Thomson E, Khoury MJ, et al. Hereditary hemochromatosis gene discovery and its implications for population-based screening. JAMA. 1998;280:172-8.

3. Steinberg KK, Cogswell ME, Chang JC, et al. Prevalence of C282Y and H63D mutations in the hemochromatosis (HFE) gene in the United States. JAMA. 2001:285:2216-22.

4. Beutler E, Felitti VJ, Koziol JA, Ho NJ, Gelbart T. Penetrance of 845G->A (C282Y) HFE hereditary haemochromatosis mutation in the USA. Lancet. 2002;359:211-8.

5. Grosse SD, Gurrin LC, Bertalli NNA, Allen KJ. Clinical penetrance in hereditary hemochromatosis: estimates of the cumulative incidence of severe liver disease among HFE C282Y homozygotes. Genet Med E-pub ahead of print 3 August 2017.

6. Bacon BR, Adams PC, Kowdley KV, Powell LW, Tavill AS; American Association for the Study of Liver Diseases. Diagnosis and management of hemochromatosis: 2011 practice guideline by the American Association for the Study of Liver Diseases. Hepatology. 2011;54: 328-43.
7. Vanclooster A, Cassiman D, Van Steenbergen W, et al. The quality of hereditary haemochromatosis guidelines: a comparative analysis. Clin Res Hepatol Gastroenterol. 2015:39:205-14.

8. de Graaff B, Neil A, Sanderson K, Si L, Yee KC, Palmer AJ. A systematic review and narrative synthesis of health economic studies conducted for hereditary haemochromatosis. Appl Health Econ Health Policy. 2015;13: 469-83.

9. Delatycki $M B$, Allen $\mathrm{KJ}$, Nisselle $A E$, et al. Use of community genetic screening to prevent HFE-associated hereditary haemochromatosis. Lancet. 2005:366:314-6.

10. Green RC, Berg JS, Grody WW, et al.; American College of Medical Genetics and Genomics. ACMG recommendations for reporting of incidental findings in clinical exome and genome sequencing. Genet Med. 2013;15:565-74.

11. Kalia SS, Adelman K, Bale SJ, et al. Recommendations for reporting of secondary findings in clinical exome and genome sequencing, 2016 update (ACMG SF v2.0): a policy statement of the American College of Medical Genetics and Genomics. Genet Med. 2017;19:249-255.

12. Adams P, Barton JC, McLaren GD, et al. Screening for iron overload: lessons from the Hemochromatosis and Iron Overload Screening (HEIRS) study. Can J Gastroenterol. 2009;23:769-72.

13. Fullerton SM, Wolf WA, Brothers KB, et al. Return of individual research results from genome-wide association studies: experience of the Electronic Medical Records and Genomics (eMERGE) Network. Genet Med. 2012;14:424-31.

14. Harrison HF, Harrison BW, Walker AP, et al. Screening for hemochromatosis and iron overload: satisfaction with results notification and understanding of mailed results in unaffected participants of the HEIRS study. Genet Test. 2008;12:491-500.

15. Gallego CJ, Burt A, Sundaresan AS, et al. Penetrance of hemochromatosis in HFE genotypes resulting in p.Cys282Tyr and $p$. [Cys282Tyr];[His63Asp] in the eMERGE Network. Am J Hum Genet. 2015;97:512-20. 